Supporting information for

\title{
Biosynthesis of the High-value Plant Secondary Product Benzyl Isothiocyanate via Functional Expression of Multiple Heterologous Enzymes in E. coli
}

Feixia Liu ${ }^{\text {a,b }}$ E-mail: feixia0909@126.com

Han Yang ${ }^{\mathrm{a}, \mathrm{b}} \quad$ E-mail: yanghan@im.ac.cn

Limin Wang ${ }^{\mathrm{a}} \quad$ E-mail: wanglimin@im.ac.cn

Bo Yu ${ }^{\text {a,** }} \quad$ E-mail: yub@im.ac.cn

Affiliations:

${ }^{a}$ CAS Key Laboratory of Microbial Physiological and Metabolic Engineering, Institute of Microbiology, Chinese Academy of Sciences, Beijing 100101, China

${ }^{b}$ University of Chinese Academy of Sciences, Beijing 100049, China

* Corresponding author.

Phone/Fax: +86-10-64806132 


\section{Supplementary Methods}

The procedures for measurement of C-S lyase (MetC) activity

The catalytic assay mixture included appropriate concentration of purified enzyme, $100 \mathrm{mM}$ phosphate buffer ( $\mathrm{pH} 7.0)$, and djenkolic acid (2 mM) as a substrate. The reaction mixture was incubated at $37^{\circ} \mathrm{C}$ for $10 \mathrm{~min}$ by adding $\mathrm{HCl}$ to terminate the reaction mixture. The assay product was analyzed by HPLC (Agilent 1260 series) equipped with Aminex HPX-87H column (Bio-Rad). The detection parameters was set as follows: $6 \mathrm{mM} \mathrm{H}_{2} \mathrm{SO}_{4}$; wavelength: $210 \mathrm{~nm}$; column temperature: $55^{\circ} \mathrm{C}$; flow rate: $0.5 \mathrm{~mL} / \mathrm{min}$; injection volume: $5 \mu \mathrm{L}$.

\section{Reference:}

Mikkelsen, M.-D., Naur, P., and Halkier, B.-A. (2004) Arabidopsis mutants in the C-S lyase of glucosinolate biosynthesis establish a critical role for indole-3-acetaldoxime in auxin homeostasis. Plant J. 37, 770-777. 


\section{Supplementary Figures}

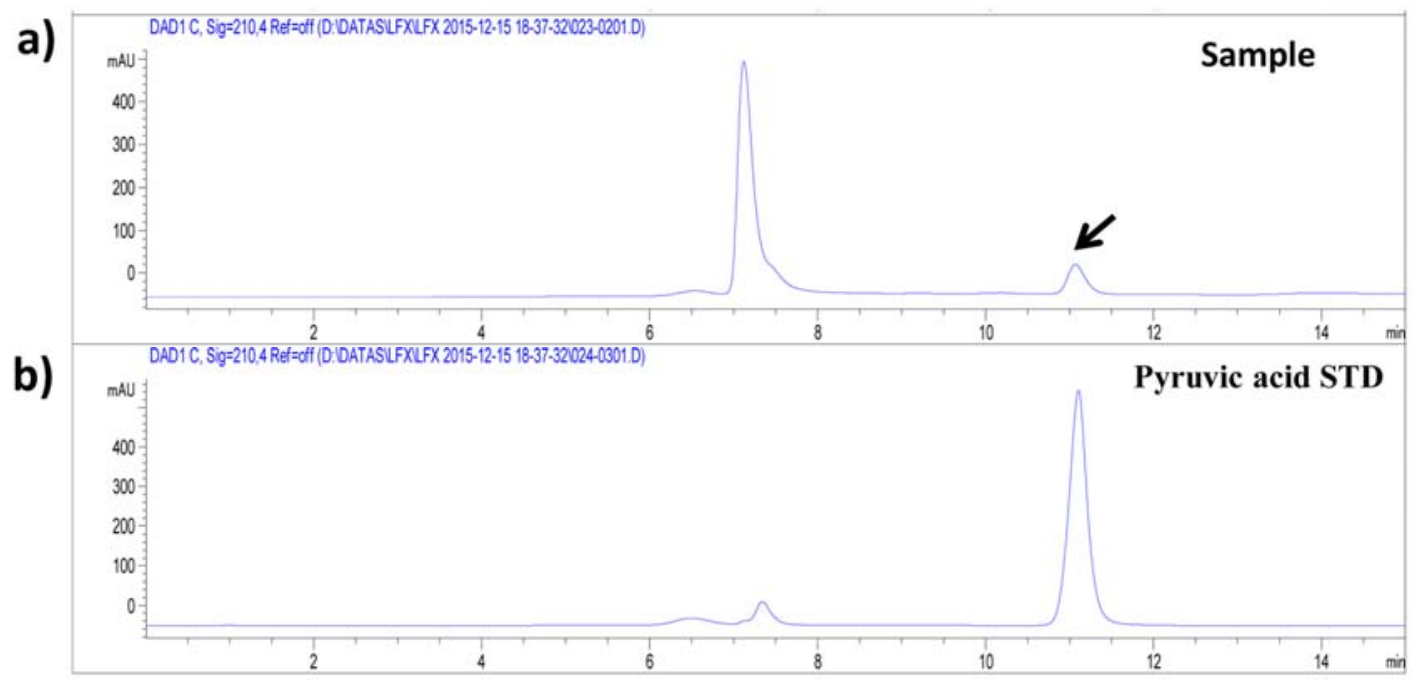

Figure S1. The functional verification of cystathionine $\beta$-lyase (MetC) from E. coli.

a) HPLC analysis of the product from djenkolic acid catalyzed by MetC and the peak of product pyruvate was indicated by arrow.

b) HPLC chromatogram of authentic standard pyruvic acid. 


\section{UGT74B1 M}

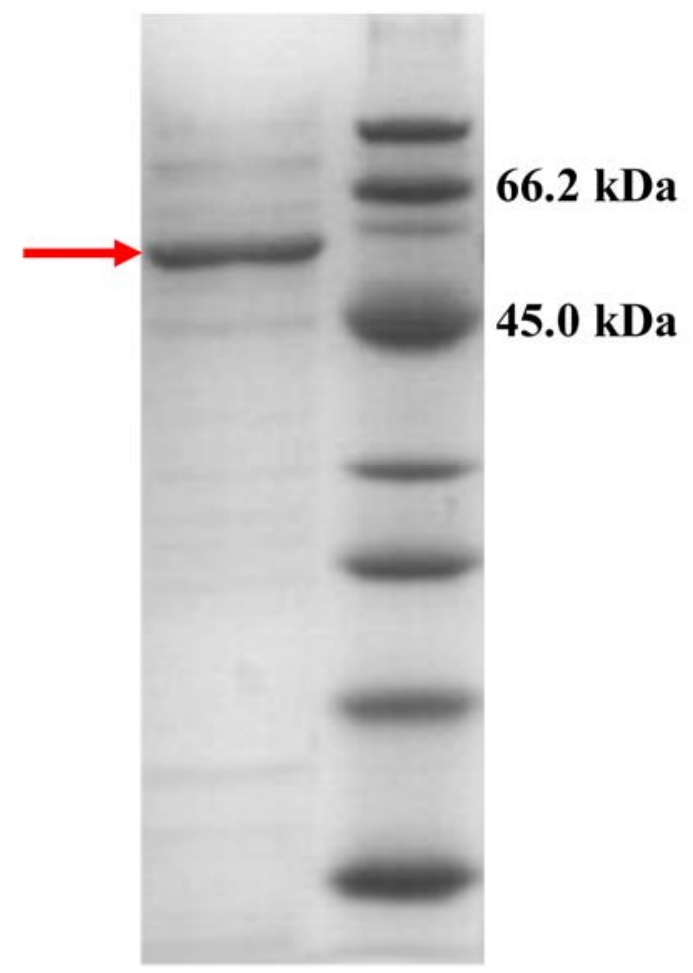

Figure S2. SDS-PAGE analysis of the purified UDPG:thiohydroximate S-glucosyltransferase from Broccoli rapa (UGT74B1)

The band of UGT74B1 was arrowed. 


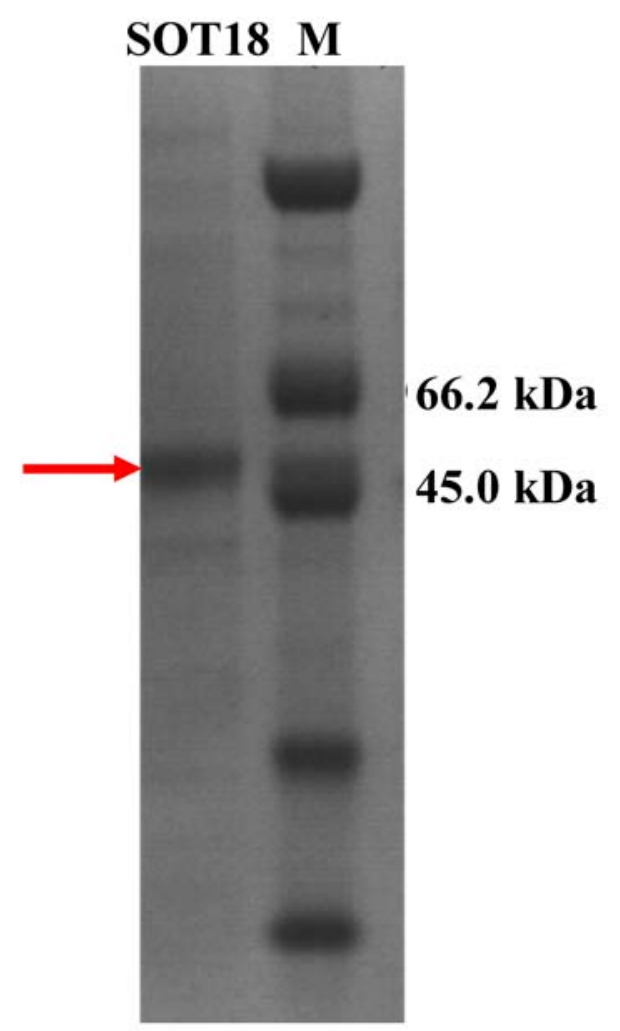

Figure S3. SDS-PAGE analysis of the purified desulfoglucosinolate: PAPS sulfotransferase from Arabidopsis thaliana ecotype Col-0 (SOT18) The band of SOT18 was arrowed. 